\title{
Effects of Late Sowing on Differentiation and Degeneration of Branches and Spikelets of Indica Rice under Different Ecological Conditions
}

\author{
Qiuping Li ${ }^{1,2 *}$, Xiaoyuan Zhong ${ }^{1,2 *}$, Fan Yang1,2, Lianhua He ${ }^{1,2}$, Yuling Zeng',2, \\ Hong Chen1,2, Li Wang1, Wanjun Ren ${ }^{1,2 \#, ~ F e i ~ D e n g 1,2 \# ~}$
}

${ }^{1}$ Key Laboratory of Crop Ecophysiology and Farming System in Southwest China of Ministry of Agriculture, Sichuan Agricultural University, Chengdu, China

${ }^{2}$ Crop Ecophysiology and Cultivation, Key Laboratory of Sichuan Province, Chengdu, China

Email: "rwjun@126.com, ${ }^{\#}$ ddf273634096@163.com

How to cite this paper: Li, Q.P., Zhong, X.Y., Yang, F., He, L.H., Zeng, Y.L., Chen, H., Wang, L., Ren, W.J. and Deng, F. (2020) Effects of Late Sowing on Differentiation and Degeneration of Branches and Spikelets of Indica Rice under Different Ecological Conditions. Agricultural Sciences, 11, 124-142.

https://doi.org/10.4236/as.2020.112008

Received: December 21, 2019

Accepted: February 4, 2020

Published: February 7, 2020

Copyright $\odot 2020$ by author(s) and Scientific Research Publishing Inc. This work is licensed under the Creative Commons Attribution International License (CC BY 4.0).

http://creativecommons.org/licenses/by/4.0/

\begin{abstract}
In rice (Oryza sativa L.), yield is related to characteristics of branches and spikelets. To investigate the effects of late sowing date on differentiation and degeneration of spikelets in rice, field experiments were conducted in Chongzhou and Hanyuan, China. Differentiation and survival of branches and spikelets in Hanyuan were lower than that of Chongzhou, whereas degeneration was greater than that of Chongzhou. In Chongzhou, sowing date affected differentiation and survival of primary, secondary, and total branches, as well as differentiation and degeneration of secondary and total spikelets. In Hanyuan, sowing date affected degeneration of secondary and total branches, and the survival and degeneration of primary spikelets. Late-sown plants experienced higher temperatures in the jointing to heading period, and there were higher sunshine hours. Rainfall and humidity were higher in Chongzhou, but lower in Hanyuan. Late sowing increased differentiation, degeneration, and survival number of branches and spikelets in Chongzhou. However, in Hanyuan, late sowing increased differentiation and degeneration of branches, but decreased survival of branches, which reduced spikelet differentiation and degeneration, resulting in fewer branches. Thus, the key to higher yield in Chongzhou was to increase differentiation of spikelets, whereas in Hanyuan, it was to reduce degeneration of branches and spikelets.
\end{abstract}

\section{Keywords}

Spikelet Differentiation, Spikelet Degeneration, Sowing Date, Rice

*These authors contributed equally to this work. 


\section{Introduction}

As one of the three major global cereal crops, rice (Oryza sativa L.) is the most important and oldest food species in the genus Oryza. Nearly half of the world's population, including most people living in East and Southeast Asia, eat rice as a daily staple [1]. According to the UN, the world's population will reach 9.6 billion by 2050. If global population growth continues at its current rate without any change in food productivity and dietary preferences, there will be severe food shortages [2] [3]. China is a large agricultural country with a large population. With the growth of the population, China will need to produce about $20 \%$ more rice by 2030 in order to meet its domestic needs if rice consumption per capita stays at the current level [4]. Therefore, increasing rice yield per unit area is a priority.

A complex correlation exists between rice grain yield, the number of panicles, spikelets per panicle, spikelet filling efficiency, and 1000-grain weight [5] [6]. The number of spikelets per panicle is an important factor in the final grain yield. Degeneration of the rice panicle leads to a reduction of grains per plant, which seriously affects the yield outcome in rice [7]. Choice of cultivar [8], fertilizer management [9], environmental conditions [10] [11], and cultural practices [12] all affect spikelet differentiation and degeneration. Differences exist in the characteristics of branches and spikelets when different planting methods are used [13]. Zhang et al. [14] found that differentiated spikelet number per panicle in higher order panicles, which had relatively larger panicles, was much larger at low planting density than at high planting density, although differentiated spikelet number per panicle in lower order panicles was similar in both planting densities.

Sowing date is one of the most important factors influencing grain yield of crops where growth duration is important. $\mathrm{Xu}$ [15] found that with delayed sowing date, rice plants experienced higher daily average temperature, the growth period was shortened (notably, the vegetative growth period), the number of grains per panicle decreased, and yield decreased. Pal et al. [16] showed that as sowing date was postponed, nitrogen accumulation and translocation, and dry matter accumulation decreased. Bashir et al. [17] showed that with the postponement of sowing date, the growth and decline of tillers in the population was uneven. Sowing time affected the differentiation and degeneration of rice spikelets and branches in an indica-japonica rice hybrid; early sowing reduced degeneration of spikelets, and thereby increased yield [18]. However, delayed sowing time has become a common phenomenon in rice production because of double-cropping and mechanized planting and transplanting in areas such as the Sichuan basin [19]. In this region, indica rice is the main type grown. The effects of delayed sowing on spikelet production of indica rice are unclear, and further research is needed.

The aim of this research was to investigate the effects of delayed sowing dates 
on various yield parameters in indica rice grown under different ecological conditions. Experimental parameters consisted of two different sowing dates and four indica rice varieties. Experiments were conducted at two sites experiencing different ecological conditions. The objectives of this study were to: 1) analyze the characteristics of spikelets at the two sites; 2) explore the effect of sowing date on the differentiation and degeneration of spikelets of different varieties; and 3) to determine the underlying causes for reduced differentiation and degeneration of spikelets. Finally, we discuss recommendations for improving cultivation conditions for indica rice.

\section{Materials and Methods}

\subsection{Study Sites and Plants}

The field experiments were conducted at Chongzhou $\left(30^{\circ} 33^{\prime} \mathrm{N}, 103^{\circ} 38^{\prime} \mathrm{E}\right)$ and Hanyuan $\left(29^{\circ} 29^{\prime} \mathrm{N}, 102^{\circ} 37^{\prime} \mathrm{E}\right)$, Sichuan, China, in 2017 . The meteorological conditions from sowing to harvest of the experimental sites were recorded (Figure 1). Topsoil characteristics of the study sites were determined (Table 1). The plants used were two hybrid indica rice combinations: Fyou-498 (F32A $\times$ Shuhui498) and Yixiangyou-2115 (Yixiang1A $\times$ Yahui2115), and two conventional indica rice cultivars: Huanghuazhan and Guichao-II.

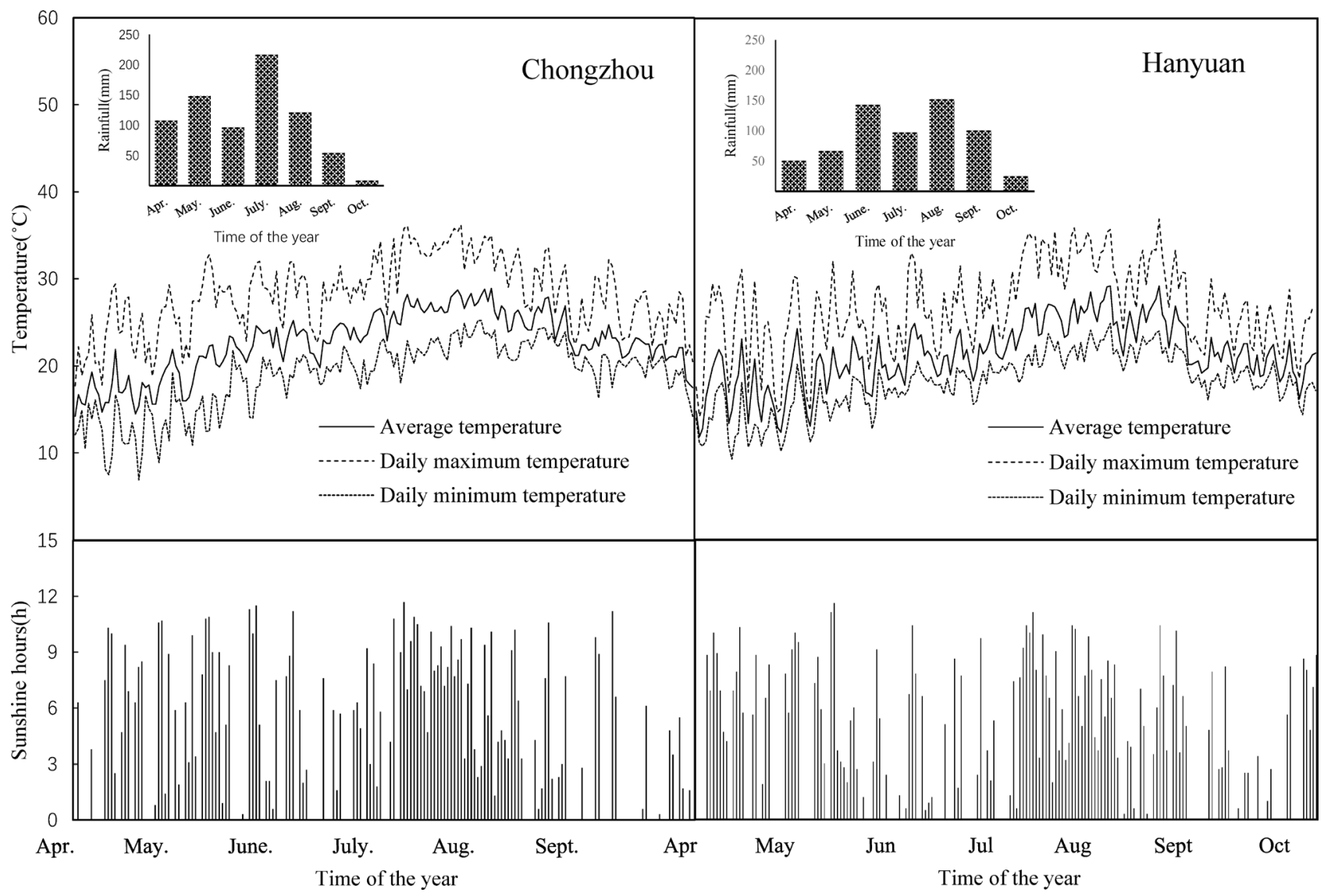

Figure 1. Climate data during the experimental period at experimental sites Chongzhou and Hanyuan, China. 
Table 1. Topsoil properties in the experimental sites in Chongzhou and Hanyuan.

\begin{tabular}{cccccccc}
\hline \multirow{2}{*}{ Site } & Organic matter & Total N & Total P & Total K & Available N & Olsen-P & Available K \\
\cline { 2 - 8 } & \multicolumn{3}{c}{$(\mathrm{g} / \mathrm{kg})$} \\
\hline Chongzhou & 29.26 & 1.85 & 7.53 & 24.85 & 2.93 & 104.25 & 102.93 \\
Hanyuan & 30.61 & 2.12 & 5.74 & 24.56 & 2.77 & 183.65 & 195.14 \\
\hline
\end{tabular}

\subsection{Experimental Design}

Experiments were conducted using a split-plot experimental design of two factors with three replications. The main area factor was sowing date (S). There were two sowing dates: normal sowing date (S1) and late sowing date (S2). The sowing dates were April $3^{\text {rd }}$ and May $3^{\text {rd }}$ for S1 and S2 at Chongzhou, respectively, and March $30^{\text {th }}$ and April $29^{\text {th }}$ for S1 and S2 at Hanyuan, respectively. The sub-area factor was four cultivars/hybrids (V), which were Fyou-498 (V1), Yixiangyou-2115 (V2), Huanghuazhan (V3) and Guichao-II (V4). The dimensions of each plot were $3.6 \mathrm{~m} \times 7 \mathrm{~m}$. The 30 -day-old seedlings were transplanted at a spacing of $30 \mathrm{~cm} \times 20 \mathrm{~cm}$ with three plants per mound. The main plot was separated by low, $0.3 \mathrm{~m}$-wide banks inserted into the soil to a depth of $0.3 \mathrm{~m}$ to avoid fertilizer and water channeling. Appropriate pesticides were used to control insects, diseases, and weeds. Water management was conducted using high-efficiency irrigation. Fertilizers used were urea for nitrogen $(\mathrm{N})$, superphosphate for phosphate $(\mathrm{P})$, and potassium chloride for potassium $(\mathrm{K})$ at rates of $180 \mathrm{~kg} / \mathrm{ha}, 90$ $\mathrm{kg} / \mathrm{ha}$ and $180 \mathrm{~kg} / \mathrm{ha}$, respectively. Nitrogen was split-applied: $75.6 \mathrm{~kg} / \mathrm{ha}$ on basal, $32.4 \mathrm{~kg} / \mathrm{ha}$ at mid-tillering, and $72.0 \mathrm{~kg} / \mathrm{ha}$ at panicle initiation. Phosphate was applied at basal. Potassium was split equally at basal and panicle initiation.

\subsection{Sampling and Measurement}

Meteorological data in the test field, including daily average temperature, daily average maximum temperature, daily average minimum temperature, sunshine duration, humidity, and rainfall during the test period, were collected by an Agroforestry Microclimate Automatic Weather Station JZ-HB (Jinzhou Sunshine Meteorological Technology Co. Ltd. China).

At the heading stage, three mounds were sampled from each sub-plot according to the average stem tiller method. Differentiation and degeneration of primary (secondary) branches and spikelets per panicle were counted. Spikelets directly attached at the primary branch were referred to as primary spikelets, and those directly attached at the secondary branch were referred to as secondary spikelets. The number of primary (secondary) branch (spikelet) differentiation was the sum of its survival and degeneration.

The methods described by Tian et al. [13], Kato et al. [20] and Ansari et al. [21] were used to calculate Branches Retrograded Percentage (BRP), Spikelets Retrograded Percentage (SRP), Primary Branches Retrograded Percentage 
(PBRP), Primary Spikelets Retrograded Percentage (PSRP), Secondary Branches Retrograded Percentage (SBRP), and Secondary Spikelets Retrograded Percentage (SSRP) as follows:

$$
\begin{aligned}
& \operatorname{BRP}(\%)=\frac{\text { Branches retrogressions }}{\text { Branches differentiations }} \times 100 \\
& \operatorname{SRP}(\%)=\frac{\text { Spikelets retrogressions }}{\text { Spikelets differentiations }} \times 100 \\
& \operatorname{PBRP}(\%)=\frac{\text { Primary branches retrogressions }}{\text { Primary branches differentiations }} \times 100 \\
& \operatorname{PSRP}(\%)=\frac{\text { Primary spikelets retrogressions }}{\text { Primary spikelets differentiations }} \times 100 \\
& \operatorname{SBRP}(\%)=\frac{\text { Secondary branches retrogressions }}{\text { Secondary branches differentiations }} \times 100 \\
& \operatorname{SSRP}(\%)=\frac{\text { Secondary spikelets retrogressions }}{\text { Secondary spikelets differentiations }} \times 100
\end{aligned}
$$

Yield was measured at each plot (adjusted to a moisture content of 14\% fresh weight). The day before harvest, the yield formations from each plot were determined by sampling five randomly-chosen mounds. Panicle-related data were recorded before threshing. Filled and unfilled spikelets were separated using a seed air separator CFY-II (Zhejiang Top Instrument Co. Ltd. China), before being counted and weighed to estimate spikelets per panicle, spikelet filling, and grain weight.

\subsection{Data Analysis}

Analysis of variance was performed using the SPSS 18.0 (SPSS Inc., Chicago, IL, USA). For the analysis, sowing date and variety were considered to be fixed effects, and the replicates were considered to be random effects. The least significant difference (LSD) test at the 0.05 level was used to determine the difference between the means of each treatment.

\section{Results}

\subsection{Analysis of Meteorological Conditions}

Accumulated temperatures, total rainfall and sunshine duration from transplanting to the heading stage of two sowing dates were recorded (Table 2). Compared to Chongzhou, decreases in the maximum and minimum temperature, humidity, and sunshine duration from transplanting to jointing of $9 \%, 2 \%$, $6 \%$, and $6 \%$, respectively, occurred in Hanyuan. Similarly, decreases in these parameters from the jointing to heading stage were $10 \%, 12 \%, 21 \%$, and $16 \%$, respectively. Accumulated temperatures followed the same trend as total rainfall. Those in Chongzhou were lower than those in Hanyuan from transplanting to the jointing stage, while from jointing to heading stage, those in Chongzhou are 
Table 2. Climate data during the transplanting to heading stage at Chongzhou and Hanyuan, China.

\begin{tabular}{|c|c|c|c|c|c|c|c|c|c|c|c|c|}
\hline \multirow[t]{2}{*}{ Treatment } & \multicolumn{2}{|c|}{$\begin{array}{c}\text { Maximum } \\
\text { Temperature }\left({ }^{\circ} \mathrm{C}\right)\end{array}$} & \multicolumn{2}{|c|}{$\begin{array}{c}\text { Minimum } \\
\text { Temperature }\left({ }^{\circ} \mathrm{C}\right)\end{array}$} & \multicolumn{2}{|c|}{$\begin{array}{c}\text { Accumulated } \\
\text { Temperature }\left({ }^{\circ} \mathrm{C}\right)\end{array}$} & \multicolumn{2}{|c|}{$\begin{array}{l}\text { Total Rainfall } \\
(\mathrm{mm})\end{array}$} & \multicolumn{2}{|c|}{ Humidity (\%RH) } & \multicolumn{2}{|c|}{$\begin{array}{c}\text { Sunshine Duration } \\
\text { (h) }\end{array}$} \\
\hline & T-J & $\mathrm{J}-\mathrm{H}$ & T-J & $\mathrm{J}-\mathrm{H}$ & T-J & $\mathrm{J}-\mathrm{H}$ & T-J & $\mathrm{J}-\mathrm{H}$ & T-J & $\mathrm{J}-\mathrm{H}$ & T-J & $\mathrm{J}-\mathrm{H}$ \\
\hline \multicolumn{13}{|l|}{ Chongzhou } \\
\hline S1V1 & 28.15 & 31.92 & 18.26 & 21.80 & 808.40 & 922.17 & 97.50 & 147.10 & 81.75 & 81.74 & 4.01 & 5.33 \\
\hline S1V2 & 27.78 & 32.85 & 17.66 & 22.11 & 1117.70 & 967.34 & 115.30 & 221.40 & 82.06 & 81.73 & 4.05 & 6.26 \\
\hline S1V3 & 27.69 & 32.15 & 17.53 & 21.86 & 1068.00 & 821.28 & 114.80 & 134.60 & 81.82 & 81.26 & 4.10 & 5.53 \\
\hline S1V4 & 27.80 & 32.60 & 17.74 & 21.88 & 1142.00 & 826.95 & 115.30 & 175.30 & 82.25 & 81.51 & 3.97 & 5.86 \\
\hline S2V1 & 28.92 & 34.53 & 19.89 & 22.96 & 852.90 & 981.16 & 88.40 & 339.30 & 85.17 & 83.02 & 3.53 & 7.11 \\
\hline S2V2 & 29.37 & 33.97 & 19.97 & 23.06 & 957.90 & 998.79 & 88.40 & 392.00 & 84.70 & 84.28 & 3.87 & 6.64 \\
\hline S2V3 & 29.37 & 34.62 & 19.97 & 23.22 & 957.90 & 941.92 & 88.40 & 220.90 & 84.70 & 83.74 & 3.87 & 7.13 \\
\hline S2V4 & 29.37 & 34.52 & 19.97 & 23.22 & 957.90 & 816.35 & 88.40 & 339.20 & 84.70 & 83.84 & 3.87 & 7.01 \\
\hline Mean & 28.55 & 33.39 & 18.87 & 22.52 & 982.84 & 909.49 & 99.56 & 246.23 & 83.39 & 82.64 & 3.91 & 6.36 \\
\hline \multicolumn{13}{|l|}{ Hanyuan } \\
\hline S1V1 & 25.30 & 31.77 & 16.73 & 20.74 & 996.80 & 979.38 & 156.10 & 159.40 & 72.10 & 73.69 & 3.33 & 4.68 \\
\hline S1V2 & 25.53 & 32.70 & 16.91 & 21.11 & 1108.00 & 1001.65 & 187.30 & 121.60 & 72.68 & 72.56 & 3.35 & 5.17 \\
\hline S1V3 & 25.50 & 32.44 & 16.94 & 20.92 & 1127.90 & 865.79 & 197.30 & 108.00 & 72.93 & 72.66 & 3.29 & 5.04 \\
\hline S1V4 & 25.50 & 32.44 & 16.94 & 20.92 & 1127.90 & 865.79 & 197.30 & 108.00 & 72.93 & 72.66 & 3.29 & 5.04 \\
\hline S2V1 & 26.13 & 32.74 & 18.35 & 21.01 & 849.90 & 870.87 & 168.70 & 98.90 & 78.12 & 72.50 & 2.29 & 6.28 \\
\hline S2V2 & 26.92 & 33.05 & 18.50 & 21.22 & 976.20 & 878.99 & 170.70 & 93.70 & 76.37 & 72.11 & 3.09 & 5.58 \\
\hline S2V3 & 26.92 & 33.22 & 18.50 & 21.38 & 976.20 & 885.46 & 170.70 & 89.90 & 76.37 & 71.82 & 3.09 & 5.58 \\
\hline S2V4 & 26.92 & 33.40 & 18.50 & 21.55 & 976.20 & 890.47 & 170.70 & 98.10 & 76.37 & 71.68 & 3.09 & 5.55 \\
\hline Mean & 26.09 & 32.72 & 17.67 & 21.11 & 1017.39 & 904.80 & 177.35 & 109.70 & 74.73 & 72.46 & 3.11 & 5.37 \\
\hline
\end{tabular}

Abbreviations: T-J: Transplanting-Jointing; J-H: Jointing-Heading.

higher than those in Hanyuan.

Sowing date affected the meteorological conditions experienced by the plants.

Compared to the normal sowing date, late sowing date leads to the increase of maximum and minimum temperatures at transplanting to jointing, and from jointing to heading stages at both sites. Sunshine duration followed the same trend. From the transplanting to jointing stage, late sowing date lead to a reduction in sunshine duration by $3 \%$ to $12 \%$ and $6 \%$ to $31 \%$, respectively. From the jointing to heading stage, sunshine duration increased by $6 \%$ to $33 \%$ and 8 to $34 \%$, respectively. With the exception of cultivar Fyou- 498 at Chongzhou, late sowing date lead to an accumulated temperature decrease of $10 \%$ to $16 \%$ and 12 to $15 \%$ from transplanting to jointing stage, respectively, in the two sites. With the exception of cultivar Fyou-498 in Hanyuan, total rainfall decreased 9\% to $23 \%$ and $9 \%$ to $13 \%$ from the transplanting to jointing stage, respectively, in the two sites. They increased $64 \%$ to $131 \%$ in Chongzhou but decreased from $9 \%$ to $38 \%$ in Hanyuan from the jointing to heading stages, respectively. For humidity, 
late sowing date lead to an increase of $3 \%$ to $4 \%$ and $5 \%$ to $8 \%$ from the transplanting to jointing stage in Chongzhou and Hanyuan, respectively, and lead to an increase of $2 \%$ to $3 \%$ from the transplanting to jointing stage in Chongzhou, and a decrease of $1 \%$ to $2 \%$ from the transplanting to jointing stage in Hanyuan.

\subsection{Effects of Sowing Date on Differentiation and Degeneration of Branches}

There were significant differences in the differentiation of branches between the two sites (Table 3). At Chongzhou, the differentiated and surviving numbers of both primary and secondary branches were higher than those at Hanyuan, which contributed to increases of $16 \%$ and $11 \%$ in the differentiated and surviving numbers of total branches, respectively.

The main effect and interaction of sowing date and cultivar on differentiation and degeneration characteristics of branches varied between the two sites. At Chongzhou, the significant effects of sowing date and cultivar was on numbers of surviving and differentiated primary, secondary, and total branches, as well as the retrograded numbers of secondary and total branches. Meanwhile, the interaction of sowing date and genotype (cultivar) had a significant influence only on the surviving and differentiated numbers of secondary and total branches. Compared to the normal sowing date, $2 \%$ to $5 \%, 1 \%$ to $5 \%, 11 \%$ to $31 \%$, and $10 \%$ to $31 \%$ increases in the surviving and differentiated numbers of primary branches, and the retrograded numbers of secondary and total branches were observed in Chongzhou, respectively. With the exception of Yixiangyou-2115, late sowing date also significantly increased numbers of surviving and differentiated secondary and total branches at Chongzhou. Furthermore, even though late sowing date significantly increased degeneration of branches, it also promoted differentiation of branches, which contributed to the highest number of surviving branches in cultivar Fyou-498. At Hanyuan, the effect of genotype (cultivar) on differentiation and degeneration characteristics was significant. However, sowing date only had obvious effects on the degeneration and percentage of secondary branches, and the degeneration of total branches. Only significant interactions of sowing date and cultivar on the degeneration and percentage of secondary and total branches were recorded. Compared to the normal sowing date, with the exception of cultivar Yixiangyou-2115, late sowing date resulted in improvements of $4 \%$ to $24 \%$ and $3 \%$ to $19 \%$ in the retrogressed and retrograde secondary branches, respectively, leading to $3 \%$ to $24 \%$ increase in the degeneration of total branches at Hanyuan. These results indicated that late sowing date could improve branch differentiation at Chongzhou, but branches retrogressed at Hanyuan.

\subsection{Effects of Sowing Date on Differentiation and Degeneration of Spikelets}

It can be seen from Table 4 that survival and differentiation number of primary 
and secondary spikelets in Chongzhou were higher than those in Hanyuan, which contributed to the $21 \%$ and $17 \%$ increases in the differentiated and surviving numbers of total spikelets, respectively.

Table 3. Number of retrograde, surviving, differentiated and retrograde percentage of branches of rice in different ecological sites and at different sowing dates.

\begin{tabular}{|c|c|c|c|c|c|c|c|c|c|c|c|c|c|}
\hline \multirow{2}{*}{\multicolumn{2}{|c|}{ Treatment }} & \multicolumn{4}{|c|}{ Primary branches } & \multicolumn{4}{|c|}{ Secondary branches } & \multicolumn{4}{|c|}{ Branches } \\
\hline & & 告 & 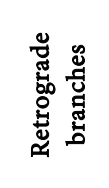 & 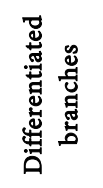 & 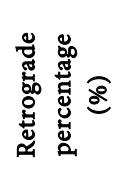 & 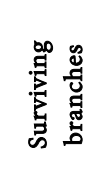 & 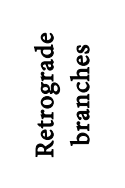 & 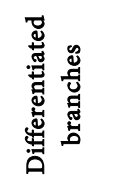 & 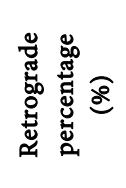 & 题 & 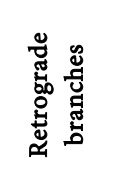 & 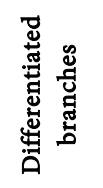 & 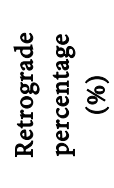 \\
\hline \multicolumn{14}{|c|}{ Chongzhou } \\
\hline S1V & & $12.47^{\mathrm{b}}$ & $0.008^{\mathrm{a}}$ & $12.48^{\mathrm{b}}$ & $0.067^{\mathrm{b}}$ & $44.88^{\mathrm{b}}$ & $18.45^{\mathrm{b}}$ & $63.33^{\mathrm{b}}$ & $29.12^{\mathrm{ab}}$ & $57.35^{\mathrm{b}}$ & $18.46^{\mathrm{b}}$ & $75.81^{\mathrm{b}}$ & $24.34^{\mathrm{ab}}$ \\
\hline S1V & & $11.53^{\mathrm{de}}$ & $0.008^{\mathrm{a}}$ & $11.54^{\mathrm{de}}$ & $0.073^{\mathrm{b}}$ & $38.30^{c}$ & $13.13^{\mathrm{c}}$ & $51.43^{\mathrm{c}}$ & $25.53^{\mathrm{b}}$ & $49.83^{c}$ & $13.14^{\mathrm{c}}$ & $62.97^{\mathrm{c}}$ & $20.86^{\mathrm{b}}$ \\
\hline S1V & & $12.18^{\mathrm{bc}}$ & $0.051^{\mathrm{a}}$ & $12.23^{\mathrm{bc}}$ & $0.421^{\mathrm{ab}}$ & $44.18^{\mathrm{b}}$ & $18.52^{\mathrm{b}}$ & $62.70^{\mathrm{b}}$ & $29.66^{\mathrm{ab}}$ & $56.36^{\mathrm{b}}$ & $18.57^{\mathrm{b}}$ & $74.93^{\mathrm{b}}$ & $24.88^{\mathrm{ab}}$ \\
\hline S1V & & $11.20^{\mathrm{e}}$ & $0.095^{\mathrm{a}}$ & $11.29^{\mathrm{f}}$ & $0.851^{\mathrm{a}}$ & $37.00^{c}$ & $16.71^{\mathrm{b}}$ & $53.71^{\mathrm{c}}$ & $31.02^{\mathrm{ab}}$ & $48.19^{c}$ & $16.80^{\mathrm{b}}$ & $65.00^{c}$ & $25.80^{\mathrm{a}}$ \\
\hline$S 2 \mathrm{~V}$ & & $13.05^{\mathrm{a}}$ & $0.056^{\mathrm{a}}$ & $13.10^{\mathrm{a}}$ & $0.427^{\mathrm{ab}}$ & $53.02^{\mathrm{a}}$ & $24.21^{\mathrm{a}}$ & $77.23^{\mathrm{a}}$ & $31.36^{\mathrm{a}}$ & $66.06^{\mathrm{a}}$ & $24.27^{\mathrm{a}}$ & 69.68 & $26.87^{\mathrm{a}}$ \\
\hline S2V & & $12.03^{\mathrm{bcd}}$ & $0.033^{\mathrm{a}}$ & $12.07^{\mathrm{bc}}$ & $0.272^{\mathrm{ab}}$ & $34.07^{\mathrm{c}}$ & $15.35^{\mathrm{bc}}$ & $49.41^{c}$ & $31.06^{\mathrm{ab}}$ & $46.10^{c}$ & $15.38^{\mathrm{bc}}$ & $90.33^{\mathrm{a}}$ & $25.02^{\mathrm{ab}}$ \\
\hline S2V & & $12.38^{\mathrm{b}}$ & $0.028^{\mathrm{a}}$ & $12.41^{\mathrm{b}}$ & $0.222^{\mathrm{ab}}$ & $51.24^{\mathrm{a}}$ & $22.38^{\mathrm{a}}$ & $73.62^{\mathrm{a}}$ & $30.38^{\mathrm{ab}}$ & $63.62^{\mathrm{a}}$ & $22.41^{\mathrm{a}}$ & $61.48^{\mathrm{c}}$ & $26.03^{\mathrm{a}}$ \\
\hline S2V & & $11.80^{\mathrm{cd}}$ & $0.074^{\mathrm{a}}$ & $11.87^{\mathrm{cd}}$ & $0.622^{\mathrm{ab}}$ & $43.67^{\mathrm{b}}$ & $18.48^{\mathrm{b}}$ & $62.15^{\mathrm{b}}$ & $29.79^{\mathrm{ab}}$ & $55.47^{\mathrm{b}}$ & $18.55^{\mathrm{b}}$ & $86.03^{\mathrm{a}}$ & $25.10^{\mathrm{ab}}$ \\
\hline Mea & & 12.08 & 0.044 & 12.12 & 0.369 & 43.29 & 18.40 & 61.70 & 29.74 & 55.37 & 18.45 & 73.82 & 24.86 \\
\hline & S & $16.98^{\star *}$ & 0.07 & $20.48^{\star *}$ & 0.05 & $14.65^{\star *}$ & $19.78^{\star *}$ & $47.93^{* *}$ & 2.36 & $16.45^{\star *}$ & $19.90^{\star *}$ & $50.51^{* *}$ & 3.17 \\
\hline \multirow[t]{2}{*}{ F-value } & $\mathrm{V}$ & $23.61^{* *}$ & 2.24 & $26.30^{* *}$ & 2.61 & $27.85^{\star *}$ & $17.52^{* *}$ & $67.28^{* *}$ & 0.68 & $29.66^{\star *}$ & $17.59^{* *}$ & $69.38^{* *}$ & 1.64 \\
\hline & $\mathrm{S} \times \mathrm{V}$ & 0.65 & 0.94 & 0.94 & 0.87 & $6.33^{* *}$ & 1.41 & $9.40^{* *}$ & 1.46 & $5.77^{\star *}$ & 1.44 & $8.76^{\star *}$ & 1.06 \\
\hline \multicolumn{14}{|c|}{ Hanyuan } \\
\hline S1V & & $11.75^{\mathrm{ab}}$ & $0.029^{c}$ & $11.78^{\mathrm{ab}}$ & $0.250^{c}$ & $44.60^{\mathrm{a}}$ & $16.92^{\mathrm{d}}$ & $61.52^{\mathrm{a}}$ & $27.54^{\mathrm{e}}$ & $56.35^{\mathrm{a}}$ & $16.95^{\mathrm{e}}$ & $73.30^{\mathrm{a}}$ & $23.15^{\mathrm{e}}$ \\
\hline S1V & & $10.20^{\mathrm{d}}$ & $0.124^{\mathrm{bc}}$ & $10.33^{\mathrm{d}}$ & $1.22^{\mathrm{bc}}$ & $28.20^{\mathrm{d}}$ & $13.65^{\mathrm{e}}$ & $41.85^{c}$ & $32.64^{\mathrm{cd}}$ & $38.41^{\mathrm{c}}$ & $13.77^{\mathrm{f}}$ & $52.18^{c}$ & $26.42^{\mathrm{cd}}$ \\
\hline S1V & & $11.20^{\mathrm{bc}}$ & $0.102^{\mathrm{bc}}$ & $11.30^{\mathrm{bc}}$ & $0.912^{\mathrm{bc}}$ & $41.05^{\mathrm{ab}}$ & $21.16^{\mathrm{b}}$ & $62.21^{\mathrm{a}}$ & $34.03^{\mathrm{bc}}$ & $52.24^{\mathrm{a}}$ & $21.26^{\mathrm{b}}$ & $73.50^{\mathrm{a}}$ & $28.94^{\mathrm{b}}$ \\
\hline S1V & & $10.54^{\mathrm{cd}}$ & $0.464^{\mathrm{a}}$ & $11.00^{c}$ & $4.24^{\mathrm{a}}$ & $34.90^{c}$ & $18.87^{\mathrm{c}}$ & $53.77^{\mathrm{b}}$ & $35.14^{\mathrm{bc}}$ & $45.44^{\mathrm{b}}$ & $19.34^{\mathrm{d}}$ & $64.78^{\mathrm{b}}$ & $29.89^{\mathrm{b}}$ \\
\hline S2V & & $11.45^{\mathrm{ab}}$ & $0.050^{c}$ & $11.50^{\mathrm{abc}}$ & $0.445^{\mathrm{bc}}$ & $42.95^{\mathrm{ab}}$ & $20.96^{\mathrm{b}}$ & $63.91^{\mathrm{a}}$ & 32.34 & $54.40^{\mathrm{a}}$ & $21.01^{b c}$ & 65.94 & $27.86^{\mathrm{bc}}$ \\
\hline S2V & & $10.00^{\mathrm{d}}$ & $0.061^{b c}$ & $10.06^{\mathrm{d}}$ & $0.614^{\mathrm{bc}}$ & $29.05^{\mathrm{d}}$ & $12.40^{\mathrm{f}}$ & $41.46^{\mathrm{c}}$ & $32.79^{\mathrm{cd}}$ & $39.05^{c}$ & $12.47^{\mathrm{g}}$ & $75.41^{\mathrm{a}}$ & $24.28^{\mathrm{de}}$ \\
\hline S2V & & $11.93^{\mathrm{a}}$ & $0.075^{b c}$ & $12.00^{\mathrm{a}}$ & $0.624^{\mathrm{bc}}$ & $39.56^{\mathrm{b}}$ & $24.96^{\mathrm{a}}$ & $64.52^{\mathrm{a}}$ & $30.04^{\mathrm{de}}$ & $51.49^{\mathrm{a}}$ & $25.03^{\mathrm{a}}$ & $51.51^{\mathrm{c}}$ & $32.74^{\mathrm{a}}$ \\
\hline S2V & & $11.20^{\mathrm{bc}}$ & $0.253^{\mathrm{b}}$ & $11.45^{\mathrm{abc}}$ & $2.23^{\mathrm{b}}$ & $34.91^{c}$ & $19.61^{c}$ & $54.52^{\mathrm{b}}$ & $38.74^{\mathrm{a}}$ & $46.10^{\mathrm{b}}$ & $19.86^{\mathrm{cd}}$ & $76.52^{\mathrm{a}}$ & $30.12^{\mathrm{b}}$ \\
\hline Mea & & 11.03 & 0.145 & 11.18 & 1.32 & 36.90 & 18.57 & 55.47 & 33.37 & 47.93 & 18.71 & 66.64 & 27.92 \\
\hline & S & 2.14 & 2.45 & 1.50 & 2.67 & 0.30 & $32.13^{\star *}$ & 1.17 & $8.48^{\star}$ & 0.099 & $29.12^{\star \star}$ & 1.32 & $8.78^{* *}$ \\
\hline \multirow[t]{2}{*}{ F-value } & $\mathrm{V}$ & $21.95^{\star \star}$ & $10.78^{\star *}$ & $31.34^{\star *}$ & $9.85^{\star *}$ & $40.81^{\star *}$ & $164.27^{\star \star}$ & $74.80^{* \star}$ & $18.83^{* *}$ & $43.55^{\star \star}$ & $161.89^{* *}$ & $77.30^{\star *}$ & $27.12^{\star \star}$ \\
\hline & $\mathrm{S} \times \mathrm{V}$ & $3.31^{\star}$ & 1.29 & $4.22^{*}$ & 1.32 & 0.34 & $15.49^{* *}$ & 0.33 & $6.68^{\star *}$ & 0.322 & $15.79^{* *}$ & 0.41 & $8.12^{* *}$ \\
\hline
\end{tabular}

Each index represents per panicle. Values within a row followed by a different small letter are significantly different at $P<0.05$. ${ }^{*}$ Significant at the 0.01 probability level; *Significant at the 0.05 probability level. The same below. 
Table 4. Number of retrograde, surviving, differentiated and retrograde percentage of spikelets of rice in different ecological sites and different sowing dates.

\begin{tabular}{|c|c|c|c|c|c|c|c|c|c|c|c|c|c|}
\hline \multirow{2}{*}{\multicolumn{2}{|c|}{ Treatment }} & \multicolumn{4}{|c|}{ Primary spikelets } & \multicolumn{4}{|c|}{ Secondary spikelets } & \multicolumn{4}{|c|}{ Spikelets } \\
\hline & & 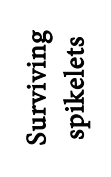 & 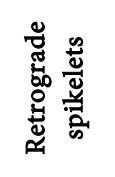 & 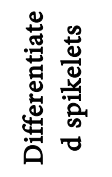 & 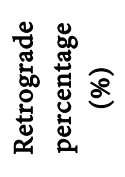 & 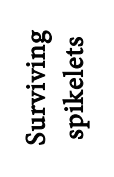 & 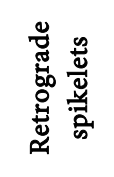 & 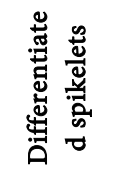 & 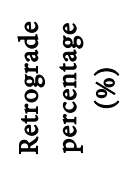 & 离造 & 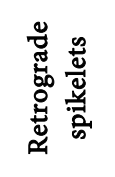 & 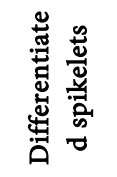 & 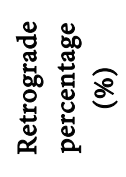 \\
\hline \multicolumn{14}{|c|}{ Chongzhou } \\
\hline $\mathrm{S} 1 \mathrm{Y}$ & & $69.73^{\mathrm{ab}}$ & $6.97^{\mathrm{ab}}$ & $76.69^{\mathrm{a}}$ & $9.10^{\mathrm{ab}}$ & $179.99^{\mathrm{b}}$ & $27.10^{\mathrm{cd}}$ & $207.09^{\mathrm{b}}$ & $13.05^{\mathrm{de}}$ & $249.71^{\mathrm{b}}$ & $34.07^{\mathrm{bcd}}$ & $283.78^{\mathrm{b}}$ & $11.98^{\mathrm{cd}}$ \\
\hline $\mathrm{S} 1$ & & $64.69^{\mathrm{cd}}$ & $1.17^{\mathrm{fg}}$ & $65.86^{c}$ & $1.77^{\mathrm{e}}$ & $141.06^{\mathrm{d}}$ & $31.13^{\mathrm{bc}}$ & $172.19^{c}$ & $18.07^{\mathrm{bc}}$ & $205.75^{\mathrm{d}}$ & $32.29^{\text {cde }}$ & $238.05^{\mathrm{cd}}$ & $13.57^{\mathrm{bcd}}$ \\
\hline $\mathrm{S} 1$ & & $63.67^{\mathrm{cd}}$ & $4.80^{\mathrm{cd}}$ & $68.47^{\mathrm{bc}}$ & $6.98^{\mathrm{bc}}$ & $155.54^{\mathrm{cd}}$ & $21.59^{d}$ & $177.14^{\mathrm{c}}$ & $12.34^{\mathrm{e}}$ & $219.21^{\mathrm{cd}}$ & $26.40^{\mathrm{de}}$ & $245.61^{\mathrm{cd}}$ & $10.79^{\mathrm{d}}$ \\
\hline S1 & & $63.79^{\mathrm{cd}}$ & $3.26^{\mathrm{de}}$ & $67.05^{c}$ & $4.90^{\mathrm{cd}}$ & $139.97^{\mathrm{d}}$ & $20.94^{\mathrm{d}}$ & $160.91^{\mathrm{cd}}$ & $12.98^{\mathrm{de}}$ & $203.76^{\mathrm{d}}$ & $24.20^{\mathrm{e}}$ & $227.95^{\mathrm{de}}$ & $10.64^{\mathrm{d}}$ \\
\hline S2 & & $70.47^{\mathrm{a}}$ & $8.56^{\mathrm{a}}$ & $79.03^{\mathrm{a}}$ & $10.83^{\mathrm{a}}$ & $206.14^{\mathrm{a}}$ & $58.71^{\mathrm{a}}$ & $264.84^{\mathrm{a}}$ & $22.26^{\mathrm{ab}}$ & $276.61^{\mathrm{a}}$ & $67.26^{\mathrm{a}}$ & $343.87^{\mathrm{a}}$ & $19.60^{\mathrm{a}}$ \\
\hline S2 & & $66.73^{\mathrm{bc}}$ & $0.89^{\mathrm{g}}$ & $67.62^{c}$ & $1.33^{\mathrm{e}}$ & $111.07^{\mathrm{e}}$ & $34.88^{\mathrm{bc}}$ & $145.95^{\mathrm{d}}$ & $23.91^{\mathrm{a}}$ & $177.81^{\mathrm{e}}$ & $35.77^{\mathrm{bc}}$ & $213.57^{\mathrm{e}}$ & $16.75^{\mathrm{ab}}$ \\
\hline S2 & & $62.67^{\mathrm{d}}$ & $6.00^{\mathrm{bc}}$ & $68.67^{\mathrm{bc}}$ & $8.74^{\mathrm{ab}}$ & $175.05^{\mathrm{bc}}$ & $35.31^{\mathrm{b}}$ & $210.36^{\mathrm{b}}$ & $16.77^{\mathrm{cd}}$ & $237.72^{\mathrm{bc}}$ & $41.31^{\mathrm{b}}$ & $279.03^{\mathrm{b}}$ & $14.78^{\mathrm{bc}}$ \\
\hline S2 & & $68.49^{\mathrm{ab}}$ & $2.78^{\mathrm{ef}}$ & $71.2^{\mathrm{b}}$ & $3.90^{\mathrm{de}}$ & $151.12^{\mathrm{d}}$ & $32.00^{\mathrm{bc}}$ & $183.12^{\mathrm{c}}$ & $17.47^{c}$ & $219.60^{\mathrm{cd}}$ & $34.78^{\mathrm{bc}}$ & $254.39^{c}$ & $13.67^{\mathrm{bcd}}$ \\
\hline $\mathrm{Me}$ & & 66.28 & 4.30 & 70.58 & 5.94 & 157.49 & 32.71 & 190.20 & 17.11 & 223.77 & 37.01 & 260.78 & 13.97 \\
\hline & S & 3.33 & 1.58 & $8.10^{*}$ & 0.76 & 1.71 & $68.01^{\star *}$ & $17.35^{\star \star}$ & $35.41^{* \star}$ & 2.68 & $70.07^{\star \star}$ & $21.52^{\star *}$ & $36.91^{* *}$ \\
\hline \multirow[t]{2}{*}{ F-value } & $\mathrm{V}$ & $10.41^{\star *}$ & $51.51^{* *}$ & $43.93^{* *}$ & $40.06^{* \star}$ & $31.13^{* *}$ & $16.15^{\star \star}$ & $41.68^{\star *}$ & $8.37^{* *}$ & $35.31^{* *}$ & $25.32^{\star *}$ & $55.69^{* *}$ & $5.85^{* *}$ \\
\hline & $\mathrm{S} \times \mathrm{V}$ & 1.83 & 1.64 & 1.23 & 1.51 & $6.05^{\star *}$ & $10.51^{\star *}$ & $11.41^{\star *}$ & 1.20 & $5,86^{* *}$ & $11.65^{\star *}$ & $11.80^{* *}$ & 2.15 \\
\hline \multicolumn{14}{|c|}{ Hanyuan } \\
\hline $\mathrm{S} 1$ & & $60.80^{\mathrm{ab}}$ & $10.79^{\mathrm{a}}$ & $71.60^{\mathrm{a}}$ & $15.06^{\mathrm{a}}$ & $177.95^{\mathrm{a}}$ & $29.68^{b}$ & $207.63^{\mathrm{a}}$ & $14.34^{\mathrm{d}}$ & $238.75^{\mathrm{a}}$ & $40.47^{\mathrm{bc}}$ & $279.23^{\mathrm{a}}$ & $14.51^{\mathrm{d}}$ \\
\hline S1 & & $57.12^{\mathrm{bc}}$ & $2.79^{\mathrm{d}}$ & $59.91^{\mathrm{d}}$ & $4.70^{\mathrm{cd}}$ & $101.71^{\mathrm{de}}$ & $37.13^{\mathrm{b}}$ & $138.58^{\mathrm{cd}}$ & $26.85^{\mathrm{a}}$ & $158.84^{\text {cd }}$ & $39.92^{\mathrm{bc}}$ & $198.49^{\mathrm{ef}}$ & $20.16^{\mathrm{abc}}$ \\
\hline S1 & & $51.27^{\mathrm{d}}$ & $7.94^{\mathrm{bc}}$ & $59.15^{\mathrm{de}}$ & $13.42^{\mathrm{a}}$ & $143.36^{\mathrm{b}}$ & $51.32^{\mathrm{a}}$ & $194.67^{\mathrm{ab}}$ & $26.30^{\mathrm{ab}}$ & $194.57^{\mathrm{b}}$ & $59.25^{\mathrm{a}}$ & $253.83^{\mathrm{bc}}$ & $23.32^{\mathrm{ab}}$ \\
\hline S1 & & $54.55^{\mathrm{cd}}$ & $7.63^{c}$ & $62.18^{\mathrm{cd}}$ & $12.29^{\mathrm{ab}}$ & $122.46^{\mathrm{c}}$ & $32.27^{b}$ & $154.73^{\mathrm{c}}$ & $20.83^{\mathrm{bc}}$ & $177.01^{\mathrm{bc}}$ & $39.90^{\mathrm{bc}}$ & $216.91^{\mathrm{de}}$ & $18.4^{\mathrm{bcd}}$ \\
\hline S2 & & $59.17^{\mathrm{abc}}$ & $9.45^{\mathrm{ab}}$ & $68.58^{\mathrm{ab}}$ & $13.79^{\mathrm{a}}$ & $167.27^{\mathrm{a}}$ & $37.37^{b}$ & $204.65^{\mathrm{a}}$ & $18.24^{\mathrm{cd}}$ & $226.40^{\mathrm{a}}$ & $46.82^{\mathrm{b}}$ & $273.22^{\mathrm{ab}}$ & $17.13^{\text {cd }}$ \\
\hline S2 & & $54.84^{\mathrm{cd}}$ & $1.01^{\mathrm{e}}$ & $55.85^{\mathrm{e}}$ & $1.83^{\mathrm{d}}$ & $100.80^{\mathrm{e}}$ & $32.01^{\mathrm{b}}$ & $132.81^{\mathrm{d}}$ & $24.27^{\mathrm{ab}}$ & $155.64^{\mathrm{d}}$ & $33.03^{c}$ & $186.66^{\mathrm{f}}$ & $17.60^{\mathrm{cd}}$ \\
\hline S2 & & $59.05^{\mathrm{abc}}$ & $6.64^{c}$ & $65.68^{\mathrm{bc}}$ & $10.13^{\mathrm{b}}$ & $130.62^{\mathrm{bc}}$ & $53.31^{\mathrm{a}}$ & $183.93^{\mathrm{b}}$ & $28.82^{\mathrm{a}}$ & $189.66^{\mathrm{b}}$ & $59.95^{\mathrm{a}}$ & $249.61^{\mathrm{c}}$ & $23.87^{\mathrm{a}}$ \\
\hline S2 & & $63.76^{\mathrm{a}}$ & $4.03^{\mathrm{d}}$ & $67.79^{\mathrm{b}}$ & $6.00^{c}$ & $120.17^{\mathrm{cd}}$ & $38.37^{b}$ & $158.54^{\mathrm{c}}$ & $24.32^{\mathrm{ab}}$ & $183.93^{\mathrm{b}}$ & $42.40^{\mathrm{bc}}$ & $226.33^{\mathrm{d}}$ & $18.76^{\mathrm{bcd}}$ \\
\hline $\mathrm{Me}$ & & 57.57 & 6.29 & 63.84 & 9.65 & 133.04 & 38.93 & 171.94 & 23.00 & 190.60 & 45.22 & 235.54 & 19.22 \\
\hline & S & $9.61^{* *}$ & $25.46^{* *}$ & 2.15 & $26.36^{* *}$ & 2.49 & 0.92 & 0.70 & 1.55 & 0.58 & 0.06 & 0.30 & 0.049 \\
\hline \multirow[t]{2}{*}{ F-value } & $\mathrm{V}$ & $5.00^{*}$ & $74.12^{* *}$ & $39.81^{* *}$ & $50.97^{* *}$ & $51.07^{* *}$ & $10.40^{* *}$ & $45.64^{* *}$ & $11.12^{* *}$ & $50.12^{* *}$ & $12.87^{* *}$ & $54.50^{* *}$ & $8.74^{\star *}$ \\
\hline & $\mathrm{S} \times \mathrm{V}$ & $8.33^{\star *}$ & 1.86 & $11.97^{* *}$ & 2.45 & 0.49 & 1.06 & 0.42 & 1.03 & 0.80 & 0.99 & 0.74 & 0.952 \\
\hline
\end{tabular}

The main effects and interactions of sowing date and cultivar on spikelet differentiation and degeneration were compared, and it was found that there were differences between the two sites. At Chongzhou, there was a significant effect of sowing date on the numbers of retrogressed and differentiated secondary and total spikelets. The effect of cultivar significantly influenced survival, differentia- 
tion, and degeneration of primary, secondary, and total spikelets. The interaction between sowing date and cultivar significantly influenced the surviving, differentiated, and retrograded numbers of secondary and total spikelets. Compared to the normal sowing date, $0.3 \%$ to $6 \%, 12 \%$ to $117 \%$, and $11 \%$ to $97 \%$ increases in the differentiated numbers of primary spikelets, and the retrograded numbers of secondary and total spikelets were observed in Chongzhou, respectively. Consistent with the trend of branches in Chongzhou, with the exception of cultivar Yixiangyou-2115, late sowing date also significantly increased the numbers of surviving and differentiated secondary and total spikelets. Late sowing resulted in a decrease in spikelet differentiation and an increase in degeneration of cultivar Fyou-498, eventually resulted in the lowest number of surviving spikelets. The main effect of cultivar on differentiation and degeneration characteristics of spikelets was significant in Hanyuan. However, sowing date had obvious effects only on the survival, degeneration and percentages of primary spikelets. Significant interactions between sowing date and cultivar on survival and differentiation of primary spikelets were recorded. Compared to the normal sowing date, the later sowing date leads to decreases of $12 \%$ to $64 \%$ and $8 \%$ to $61 \%$ decrease in the degeneration and retrograded percentage of primary spikelets, respectively. With the exception of cultivar Yixiangyou-2115, late sowing date resulted in $4 \%$ to $26 \%$ and $1 \%$ to $16 \%$ improvement in the degeneration of secondary and total spikelets, respectively. With the exception of cultivar Guichao II, late sowing date leads to $1 \%$ to $6 \%, 2 \%$ to $5 \%, 1 \%$ to $6 \%$, and $2 \%$ to $6 \%$ decreases in survival and differentiation of secondary and total spikelets, respectively. These results showed that late sowing could improve the differentiation and degeneration of total spikelets in Chongzhou but decreased the differentiation and survival of total spikelets in Hanyuan.

\subsection{Relationship of Branch and Spikelet Characteristics to Climatic Factors}

Correlation analysis showed that relationships between branch and spikelet traits with climatic factors varied at Chongzhou and Hanyuan. The degeneration and retrograded percentages of total and secondary spikelet numbers were significantly positively correlated with most climatic factors. At Chongzhou (Table 5), the degeneration and retrograded percentage of secondary and total spikelets were significantly positively correlated with all climatic factors. The number of retrogressed and differentiated secondary branches was significantly positively correlated with the maximum temperature and average temperature. The number of retrogressive and differentiated total branches was also significantly positively correlated with the average temperature and temperature difference.

At Hanyuan (Table 6), the maximum temperature, average temperature, temperature difference and rainfall were significantly positively correlated with the retrograded percentage of secondary, total branches and secondary spikelets. In contrast, they were significantly negatively correlated with the degeneration 
Table 5. Effect of climatic factors during jointing to heading on the branch and spikelet characteristics of rice in Chongzhou.

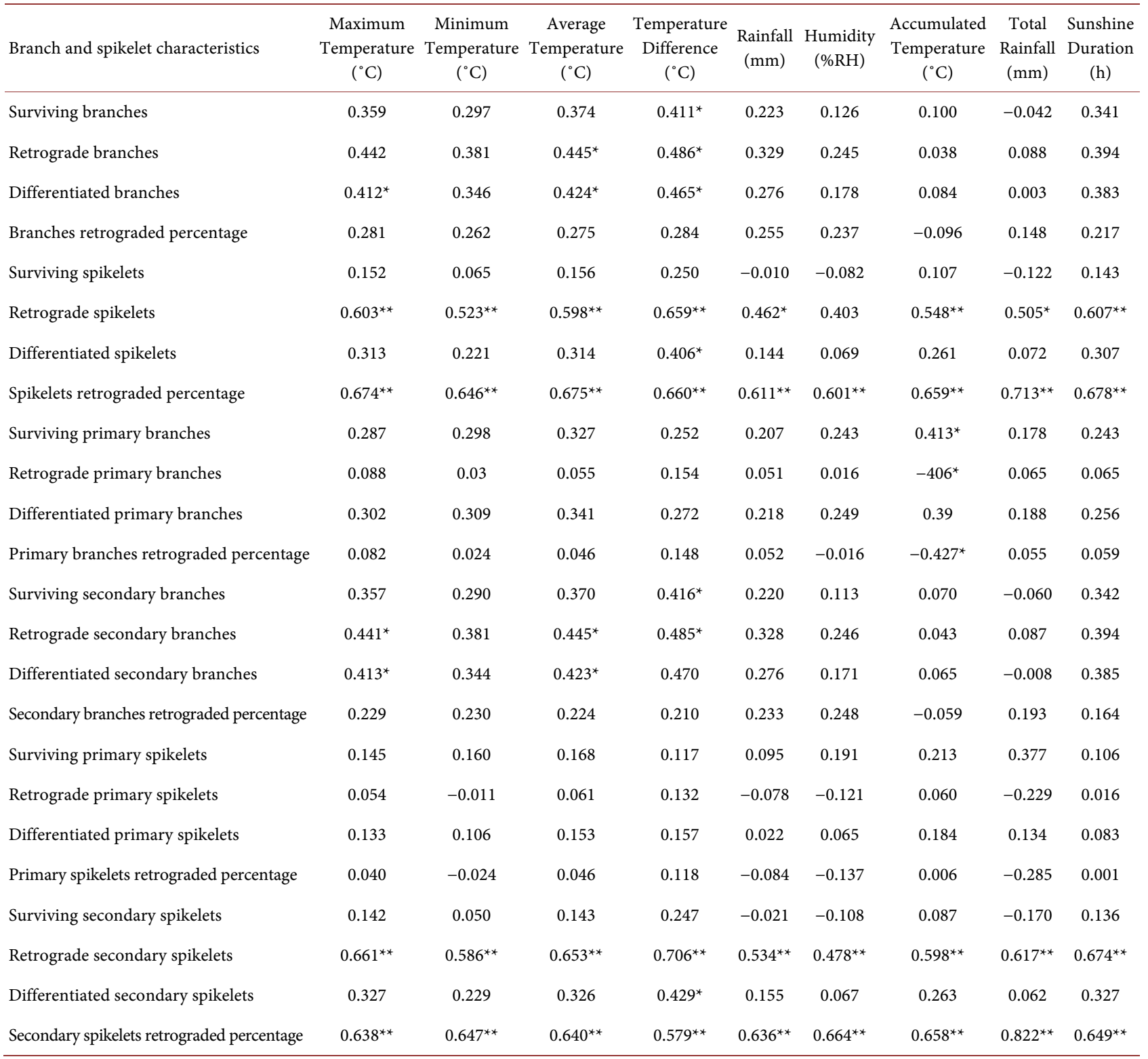

Table 6. Effect of climatic factor during jointing to heading on the branches and spikelets characters of rice in Hanyuan.

\begin{tabular}{|c|c|c|c|c|c|c|c|c|c|}
\hline Branch and spikelet characteristics & $\begin{array}{c}\text { Maximum } \\
\text { Temperature } \\
\left({ }^{\circ} \mathrm{C}\right)\end{array}$ & $\begin{array}{c}\text { Minimum } \\
\text { Temperature } \\
\left({ }^{\circ} \mathrm{C}\right)\end{array}$ & $\begin{array}{c}\text { Average } \\
\text { Temperature } \\
\left({ }^{\circ} \mathrm{C}\right)\end{array}$ & $\begin{array}{c}\text { Temperature } \\
\text { Difference } \\
\left({ }^{\circ} \mathrm{C}\right)\end{array}$ & $\begin{array}{l}\text { Rainfall } \\
(\mathrm{mm})\end{array}$ & $\begin{array}{c}\text { Humidity } \\
(\% \mathrm{RH})\end{array}$ & $\begin{array}{c}\text { Accumulated } \\
\text { Temperature } \\
\left({ }^{\circ} \mathrm{C}\right)\end{array}$ & $\begin{array}{c}\text { Total } \\
\text { Rainfall } \\
(\mathrm{mm})\end{array}$ & $\begin{array}{c}\text { Sunshine } \\
\text { Duration } \\
\text { (h) }\end{array}$ \\
\hline Surviving branches & -0.39 & 0.394 & -0.404 & -0.36 & $-0.413^{*}$ & 0.388 & -0.147 & 0.288 & -0.004 \\
\hline Retrograde branches & 0.191 & -0.229 & 0.186 & 0.178 & 0.179 & -0.256 & $-0.498^{*}$ & -0.327 & 0.239 \\
\hline Differentiated branches & -0.193 & 0.181 & -0.205 & -0.178 & -0.214 & 0.166 & -0.302 & 0.068 & 0.094 \\
\hline Branches retrograded percentage & $0.521^{\star *}$ & $0.486^{*}$ & $0.521^{\star *}$ & $0.513^{*}$ & $-0.556^{\star *}$ & $-0.600^{* *}$ & $-0.502^{*}$ & $-0.603^{\star *}$ & 0.272 \\
\hline Surviving spikelets & $0.499^{*}$ & $0.551^{* *}$ & $-0.517^{\star *}$ & $-0.455^{\star}$ & $-0.535^{\star \star}$ & $0.541^{* *}$ & 0.059 & $0.474^{*}$ & 0.002 \\
\hline Retrograde spikelets & 0.09 & -0.178 & 0.089 & 0.066 & 0.105 & -0.152 & -0.278 & -0.222 & 0.074 \\
\hline
\end{tabular}




\begin{tabular}{|c|c|c|c|c|c|c|c|c|c|}
\hline ontinued & & & & & & & & & \\
\hline Differentiated spikelets & $-0.411^{*}$ & $0.428^{*}$ & $-0.427^{*}$ & -0.380 & $-0.438^{*}$ & $0.428^{*}$ & -0.04 & 0.345 & 0.026 \\
\hline Spikelets retrograded percentage & 0.350 & 0.300 & 0.345 & 0.370 & $-0.445^{*}$ & $-0.418^{*}$ & -0.243 & $-0.434^{*}$ & 0.045 \\
\hline Surviving primary branches & -0.072 & 0.205 & -0.121 & -0.025 & -0.204 & 0.118 & -0.071 & 0.153 & 0.060 \\
\hline Retrograde primary branches & 0.068 & -0.072 & 0.068 & 0.061 & 0.070 & -0.138 & -0.248 & -0.148 & -0.175 \\
\hline Differentiated primary branches & -0.063 & 0.206 & -0.115 & -0.014 & -0.204 & 0.096 & -0.133 & 0.132 & 0.019 \\
\hline Primary branches retrograded percentage & 0.054 & -0.066 & 0.055 & 0.044 & 0.062 & -0.123 & -0.231 & -0.138 & -0.183 \\
\hline Surviving secondary branches & $-0.420^{*}$ & $0.409^{*}$ & $-0.429^{\star}$ & -0.393 & $-0.430^{*}$ & $0.413^{*}$ & -0.153 & 0.298 & -0.011 \\
\hline Retrograde secondary branches & 0.189 & -0.226 & 0.184 & 0.176 & 0.177 & -0.251 & $-0.489^{*}$ & -0.321 & 0.247 \\
\hline Differentiated secondary branches & -0.201 & 0.177 & -0.209 & -0.189 & -0.213 & 0.169 & 0.313 & 0.062 & 0.099 \\
\hline Secondary branches retrograded percentage & $0.601^{* *}$ & $-0.617^{\star \star}$ & $0.598^{* *}$ & $0.565^{\star *}$ & $0.581^{* *}$ & $-0.667^{\star *}$ & $-0.443^{*}$ & $-0.636^{\star *}$ & 0.285 \\
\hline Surviving primary spikelets & 0.298 & 0.077 & 0.220 & 0.383 & 0.045 & -0.137 & 0.269 & 0.135 & 0.209 \\
\hline Retrograde primary spikelets & $-0.633^{* *}$ & $0.564^{* *}$ & $-0.637^{\star *}$ & $-0.614^{* *}$ & $-0.609^{* *}$ & $0.607^{* *}$ & -0.050 & $0.451^{*}$ & -0.165 \\
\hline Differentiated primary spikelets & -0.159 & $0.419^{*}$ & -0.224 & -0.078 & -0.348 & 0.273 & 0.193 & 0.396 & 0.057 \\
\hline Primary spikelets retrograded percentage & $-0.631^{* *}$ & $0.517^{\star *}$ & $-0.626^{* *}$ & $-0.624^{* *}$ & $-0.577^{\star \star}$ & $0.577^{* *}$ & -0.115 & 0.392 & -0.191 \\
\hline Surviving secondary spikelets & $-0.567^{* *}$ & 0.562 & $-0.573^{* *}$ & $-0.533^{* *}$ & $-0.563^{* *}$ & $0.585^{* *}$ & 0.020 & $0.473^{*}$ & -0.031 \\
\hline Retrograde secondary spikelets & 0.304 & -0.376 & 0.304 & 0.272 & 0.312 & -0.361 & -0.281 & -0.385 & 0.133 \\
\hline Differentiated secondary spikelets & $-0.435^{*}$ & $0.407^{*}$ & $-0.441^{*}$ & $-0.414^{*}$ & $-0.431^{*}$ & $0.433^{*}$ & -0.078 & 0.318 & 0.017 \\
\hline Secondary spikelets retrograded percentage & $0.570^{\star *}$ & $-0.600^{\star *}$ & $0.569^{* *}$ & $0.530^{\star *}$ & $0.561^{* *}$ & $-0.607^{\star *}$ & -0.146 & $-0.535^{\star *}$ & 0.112 \\
\hline
\end{tabular}

and retrograded percentage of primary spikelets, the surviving and differentiated number of secondary and total spikelets. The minimum temperature, humidity and total rainfall showed a significant negative correlation with the retrograded percentage of total branches, secondary branch and spikelet, and significant positive correlation with the survivor and differentiation number of secondary and total spikelets, the degeneration and retrograded percentage of primary spikelet.

\subsection{Relationship between Branch and Spikelet Characteristics with Yield and Yield Components}

From the data presented in Table 7, it can be seen that the branch and spikelet traits in Chongzhou correlated with yield and its components. The number of spikelets per panicle in Chongzhou was significantly positively correlated with the branch and spikelet traits. The effective panicles were significantly negatively correlated with the retrogressed and retrograded percentage of secondary and total spikelets. The thousand-grain weight was significantly negatively correlated with the survival, differentiation and degeneration of secondary and total branches, the rate of degeneration, and degeneration of primary spikelets. There was no significant correlation between the yield and degeneration of branches, but there was a significant positive correlation between the yield and the differentiation and survival of spikelets, which indicates that promoting differentiation of spikelets in Chongzhou is the key to increasing yield there. 
Table 7. Effect of yield and yield components on branches and spikelets characters of rice in Chongzhou.

\begin{tabular}{|c|c|c|c|c|c|}
\hline Branch and spikelet characteristics & $\begin{array}{l}\text { Effective panicles } \\
\qquad\left(\times 10^{4} / \mathrm{ha}\right)\end{array}$ & $\begin{array}{c}\text { Number of spikelets } \\
\text { per panicle }\end{array}$ & $\begin{array}{c}\text { Setting } \\
\text { percentage (\%) }\end{array}$ & $\begin{array}{l}\text { Thousand grain } \\
\text { weight }(\mathrm{g})\end{array}$ & $\begin{array}{l}\text { Grain yield } \\
\text { (t/ha) }\end{array}$ \\
\hline Surviving branches & -0.034 & $0.795^{* *}$ & -0.076 & $-0.592^{* *}$ & 0.145 \\
\hline Retrograde branches & -0.037 & $0.710^{* *}$ & -0.075 & $-0.586^{\star *}$ & 0.010 \\
\hline Differentiated branches & -0.037 & $0.816^{* *}$ & -0.080 & $-0.628^{\star *}$ & 0.105 \\
\hline Branches retrograded percentage & 0.01 & 0.277 & -0.036 & 0.272 & -0.326 \\
\hline Surviving spikelets & -0.268 & $0.876^{* *}$ & -0.208 & -0.393 & $0.460^{*}$ \\
\hline Retrograde spikelets & $-0.527^{\star \star}$ & $0.614^{* *}$ & -0.330 & 0.022 & 0.339 \\
\hline Differentiated spikelets & -0.376 & $0.868^{* *}$ & -0.266 & -0.292 & $0.461^{*}$ \\
\hline Spikelets retrograded percentage & $-0.511^{*}$ & -0.152 & -0.296 & 0.168 & 0.262 \\
\hline Surviving primary branches & $-0.409^{*}$ & $0.778^{\star *}$ & -0.075 & -0.24 & 0.261 \\
\hline Retrograde primary branches & 0.217 & -0.186 & -0.163 & -0.109 & -0.184 \\
\hline Differentiated primary branches & -0.399 & $0.782^{* *}$ & -0.088 & -0.258 & 0.249 \\
\hline Primary branches retrograded percentage & 0.240 & -0.213 & -0.161 & -0.108 & -0.207 \\
\hline Surviving secondary branches & 0.001 & $0.779^{* *}$ & -0.074 & $-0.610^{* *}$ & 0.132 \\
\hline Retrograde secondary branches & -0.040 & $0.713^{* *}$ & -0.073 & $-0.585^{* *}$ & 0.013 \\
\hline Differentiated secondary branches & -0.015 & $0.807^{* *}$ & -0.079 & $-0.642^{* *}$ & 0.096 \\
\hline Secondary branches retrograded percentage & -0.035 & 0.152 & -0.019 & -0.191 & -0.17 \\
\hline Surviving primary spikelets & $-0.630^{\star *}$ & $0.424^{*}$ & $-0.585^{\star *}$ & 0.343 & $0.439^{*}$ \\
\hline Retrograde primary spikelets & -0.221 & $0.904^{* *}$ & -0.06 & $-0.504^{\star}$ & 0.389 \\
\hline Differentiated primary spikelets & $-0.570^{\star \star}$ & $0.815^{* *}$ & $-0.446^{*}$ & -0.047 & $0.530^{* *}$ \\
\hline Primary spikelets retrograded percentage & -0.126 & $0.871^{* *}$ & 0.003 & $-0.577^{* *}$ & 0.317 \\
\hline Surviving secondary spikelets & -0.207 & $0.864^{* *}$ & -0.149 & $-0.449^{\star}$ & $0.429^{*}$ \\
\hline Retrograde secondary spikelets & $-0.537^{* *}$ & $0.476^{*}$ & -0.355 & 0.141 & 0.288 \\
\hline Differentiated secondary spikelets & -0.339 & $0.848^{* *}$ & -0.235 & -0.316 & $0.438^{*}$ \\
\hline Secondary spikelets retrograded percentage & $-0.474^{* *}$ & -0.069 & -0.277 & $0.490^{*}$ & 0.010 \\
\hline
\end{tabular}

At Hanyuan, thousand-grain weight was significantly negatively correlated with branch and spikelet traits (Table 8). There is a significant positive correlation between the effective panicles and the retrogressed and retrograded percentage of secondary and total branches and secondary and total spikelets. The number of spikelets per panicle was significantly positively correlated with the survival and differentiation of branches and spikelets. There was a negative correlation between the yield at Hanyuan and the retrogressed and retrograded percentage of secondary and total branches and spikelets. This indicates that the key to increasing yield is to reduce degeneration of spikelets and branches by ensuring the proper amount of spikelet differentiation under the ecological conditions present at Hanyuan. 
Table 8. Effect of yield and yield components on branches and spikelets characters of rice in Hanyuan.

\begin{tabular}{|c|c|c|c|c|c|}
\hline Branch and spikelet characteristics & $\begin{array}{l}\text { Effective panicles } \\
\qquad\left(\times 10^{4} / \mathrm{ha}\right)\end{array}$ & $\begin{array}{l}\text { Number of spikelets } \\
\text { per panicle }\end{array}$ & $\begin{array}{c}\text { Setting } \\
\text { percentage (\%) }\end{array}$ & $\begin{array}{l}\text { Thousand grain } \\
\text { weight }(\mathrm{g})\end{array}$ & $\begin{array}{l}\text { Grain yield } \\
(\mathrm{t} / \mathrm{ha})\end{array}$ \\
\hline Surviving branches & 0.014 & $-0.417^{\star}$ & 0.173 & $-0.557^{\star *}$ & 0.247 \\
\hline Retrograde branches & $0.488^{*}$ & -0.154 & -0.019 & $-0.866^{* *}$ & $-0.482^{*}$ \\
\hline Differentiated branches & 0.206 & $0.661^{* *}$ & 0.112 & $-0.735^{\star *}$ & -0.024 \\
\hline Branches retrograded percentage & $0.607^{*}$ & $0.504^{\star}$ & -0.185 & -0.38 & $-0.676^{* *}$ \\
\hline Surviving spikelets & -0.300 & $0.807^{* *}$ & 0.143 & -0.271 & $0.521^{\star *}$ \\
\hline Retrograde spikelets & $0.573^{\star *}$ & -0.057 & 0.111 & $-0.746^{* *}$ & -0.364 \\
\hline Differentiated spikelets & -0.079 & $0.694^{* *}$ & 0.160 & $-0.481^{\star}$ & 0.341 \\
\hline Spikelets retrograded percentage & $0.684^{* *}$ & $0.445^{*}$ & 0.032 & $-0.490^{*}$ & $-0.525^{\star *}$ \\
\hline Surviving primary branches & 0.106 & $0.455^{*}$ & 0.061 & $-0.599^{* *}$ & 0.044 \\
\hline Retrograde primary branches & 0.233 & -0.321 & -0.224 & -0.117 & -0.314 \\
\hline Differentiated primary branches & 0.168 & $0.411^{*}$ & 0.013 & $-0.670^{* *}$ & -0.026 \\
\hline Primary branches retrograded percentage & 0.222 & -0.324 & -0.207 & -0.097 & -0.299 \\
\hline Surviving secondary branches & 0.002 & $-0.417^{\star}$ & 0.183 & $-0.541^{* *}$ & 0.267 \\
\hline Retrograde secondary branches & $0.479^{*}$ & -0.154 & -0.010 & $-0.863^{\star *}$ & $-0.470^{*}$ \\
\hline Differentiated secondary branches & 0.208 & $0.661^{* *}$ & 0.118 & $-0.734^{* *}$ & -0.023 \\
\hline Secondary branches retrograded percentage & $0.588^{* *}$ & 0.181 & -0.207 & $-0.582^{\star \star}$ & $-0.822^{\star *}$ \\
\hline Surviving primary spikelets & $-0.413^{*}$ & 0.397 & -0.399 & 0.160 & 0.180 \\
\hline Retrograde primary spikelets & -0.047 & $0.649^{* *}$ & 0.243 & $-0.506^{*}$ & 0.358 \\
\hline Differentiated primary spikelets & -0.362 & $0.718^{* *}$ & -0.163 & -0.182 & 0.365 \\
\hline Primary spikelets retrograded percentage & 0.070 & $0.548^{* *}$ & 0.267 & $-0.575^{\star *}$ & 0.267 \\
\hline Surviving secondary spikelets & -0.250 & $0.780^{* *}$ & 0.209 & -0.307 & 0.515 \\
\hline Retrograde secondary spikelets & $0.628^{* *}$ & -0.274 & 0.038 & $-0.631^{* *}$ & $-0.507^{\star}$ \\
\hline Differentiated secondary spikelets & -0.023 & $0.649^{* *}$ & 0.210 & $-0.508^{\star}$ & 0.317 \\
\hline Secondary spikelets retrograded percentage & $0.569^{* *}$ & $-0.661^{* *}$ & -0.097 & -0.222 & $-0.647^{* *}$ \\
\hline
\end{tabular}

\section{Discussion}

With the decrease of cultivated land area, efficient use of cultivated land has become a priority focus of research. The planting pattern of the double-cropping system has effectively improved land utilization rate, but it delays the sowing date of rice [22]. There has been a lot of research done on the influence of climate change on rice growth stage caused by postponing sowing date [23]. Due to the difference between experimental sites and sowing time, results vary. When sowing date is delayed, accumulated temperature and sunshine duration for growth stages are significantly reduced [24]. Xing et al. [25] and Hadgu et al. [26] found that when the sowing date was delayed, the daily average temperature during the filling and setting period of rice decreased, the daily average temper- 
ature difference increased slightly, and the daily average sunshine duration showed a downward trend. The results of this study indicated that the effects of sowing date on climate varied depending on the site and ecological conditions. Sowing date can play a critical role in optimizing the use of environmental factors like temperature during crop growth to favorably influence yield and quality characteristics [27]. In this experiment, late sowing increased the daily maximum temperature, daily minimum temperature, and sunshine duration from the jointing to heading stage. Total rainfall and humidity in Chongzhou increased, while the total rainfall and humidity were decreased in Hanyuan (Table 2).

Choice of sowing date will inevitably influence plant growth due to availability of different environmental factors such as temperature and light [17]. With a delay in rice sowing date, nitrogen accumulation and transportation, and dry matter accumulation decrease, resulting in a shorter growth period and a decrease in yield [15] [16] [28]. With a delay in sowing date, the vegetative growth period shortens, which leads to acceleration of the growth process from sowing to heading, and shortening of the growth days [29]. Delaying sowing also reduced plant height, leaf area, root biomass and tillering, and total biomass [30] [31]. It also influenced the duration of the differentiation of branch spikelets, thus affecting the formation of spikelets [29]. In the current study, plants sown late experienced an increased daily maximum temperature from the jointing to heading stages and increased the differentiation and degeneration of branches at both sites. Late sowing date increased differentiation and degeneration of spikelets in Chongzhou, whereas these effects decreased at Hanyuan. Correlation analysis revealed that degeneration and retrograded percentages of secondary and total spikelets in Chongzhou were significantly positively correlated with all meteorological factors. Retrograded percentage of primary spikelets, secondary spikelets, secondary and total branches in Hanyuan was significantly correlated with temperature, temperature difference, rainfall and humidity. Fu et al. [32] found that high temperatures decreased spikelet differentiation in rice. Our findings were similar, confirming that environmental changes influenced by sowing date affect differentiation and degeneration of spikelets and branches.

Yield is closely linked to number of spikelets per unit area, which varies with genotype and environment [33]. Decrease in the number of grains per panicle is the main factor reducing yield in rice that is sown late [34]. Late sowing, shortened the growth period of the plant which reduced the number of kernels per panicle compared to rice sown earlier, resulting in a decline in yields [17] [35]. In this experiment, the delay in sowing date resulted in a decrease in the differentiation of total spikelets in Hanyuan. This is in accordance with other research [18], which found a delay of sowing date caused a significant decrease in total spikelets per panicle, especially the differentiation of the secondary spikelet. The delay of sowing date lead to an increase in differentiation of total spikelets in Chongzhou, and the differentiation of total branches per panicle at both sites increased significantly. The number of branches and spikelets per panicle not 
only depends on the number of differentiated branches and spikelets at the early differentiation stage, but also on the number of retrogressed branches and spikelets at the late differentiation stage [36] [37]. Late sowing led to a reduction in the number of degenerations of total branches and spikelets at both sites. This resulted in an increase in the survival number of total branches and spikelets in Chongzhou, but a decrease in Hanyuan.

Zhong et al. [38] pointed out that the differentiation and formation of panicle branches and spikelets related to index characters should be improved mainly through cultivar breeding, while the degeneration-related index characters of panicle branches and spikelets are likely to be optimized through improved cultivation conditions. We found that there was no significant correlation between the yield at Chongzhou and the differentiation and degeneration of branches, but there is a significant positive correlation between the yield and the differentiation and survival of spikelets (Table 7). The differentiation and survival of total branches and total spikelets at the late sowing date was highest, but the increase of rainfall in the late stage was not conducive to grain filling and development, which resulted in a decrease in grain yield. This indicates that the key to increasing yield at Chongzhou is to increase the differentiated number of spikelets. However, there is a negative correlation between the yield in Hanyuan and the degeneration of branches and spikelets, which indicates that on the basis of spikelet differentiation, reducing the degeneration of branches and spikelets is the key to yield increase. Therefore, selection of sowing time should consider that the period of grain filling should avoid the period of high temperatures, and high rainfall, so as to achieve the maximum yield.

\section{Conclusion}

The number of spikelets per panicle is one of the most important components of rice yield, and this component was significantly influenced by ecological conditions. Our study showed that differentiation and degeneration of rice were not only affected by ecological conditions, but also by sowing date. When sowing date was delayed, the differentiation of total spikelets in Hanyuan decreased, while in Chongzhou it increased, and the differentiation of total branches per spike at both sites increased significantly. The number of retrograde branches and spikelets in both Chongzhou and Hanyuan increased, resulting in an increase in the survival number of branches and spikelets in Chongzhou, but a decrease in Hanyuan. This indicates that the key to increasing yield at Chongzhou is to increase the differentiation number of spikelets, and for Hanyuan, reducing the degeneration of branches and spikelets is the key to yield increase.

\section{Acknowledgements}

This work was supported by the National Key R \& D Program of China under Grant [number 2017YFD0300105]; Ministry of Science and Technology of the People's Republic of China under Grant [number 2016YFD0300506]; and the 
Science and Technology Department of Sichuan Province under Grant [number 2016NYZ0051]. We also thank the students and farmers involved in the experiments.

\section{Conflicts of Interest}

The authors declare no conflicts of interest regarding the publication of this paper.

\section{References}

[1] Jian, Z.P., Fei, W., Li, Z.Z., Chen, Y.T., Ma, X.C., Nie, L.X., et al. (2013) Grain Yield and Nitrogen Use Efficiency Responses to N Application in Bt (CrylAb/Ac) Transgenic Two-Line Hybrid Rice. Field Crops Research, 155, 184-191. https://doi.org/10.1016/j.fcr.2013.09.007

[2] Ray, D.K., Mueller, N.D., West, P.C. and Foley, J.A. (2013) Yield Trends Are Insufficient to Double Global Crop Production by 2050. PLoS ONE, 8, e66428.

https://doi.org/10.1371/journal.pone.0066428

[3] Wang, F. and Peng, S.B. (2017) Yield Potential and Nitrogen Use Efficiency of China's Super Rice. Journal of Integrative Agriculture, 16, 1000-1008. https://doi.org/10.1016/S2095-3119(16)61561-7

[4] Peng, S.B., Tang, Q.Y. and Zou, Y.B. (2009) Current Status and Challenges of Rice Production in China. Plant Production Science, 12, 3-8. https://doi.org/10.1626/pps.12.3

[5] You, C.C., Zhu, H.L., Xu, B.B., Huang, W.X., Wang, S.H., Ding, Y.F., et al. (2016) Effect of Removing Superior Spikelets on Grain Filling of Inferior Spikelets in Rice. Frontiers in Plant Science, 7, 1161. https://doi.org/10.3389/fpls.2016.01161

[6] Xu, Q., Chen, W.F. and Xu, Z. (2015) Relationship between Grain Yield and Quality in Rice Germplasms Grown across Different Growing Areas. Breeding Science, 65, 226-232. https://doi.org/10.1270/jsbbs.65.226

[7] Yan, W., Lu, Y.Y., Chang, Z.Y., Wang, S.H., Ding, Y.F. and Ding, C.Q. (2018) Transcriptomic Analysis of Field-Grown Rice (Oryza sativa L.) Reveals Responses to Shade Stress in Reproductive Stage. Plant Growth Regulation, 84, 1-10. https://doi.org/10.1007/s10725-017-0363-3

[8] Rang, Z.W., Jagadish, S.V.K., Zhou, Q.M., Craufurd, P.Q. and Heuer, S. (2011) Effect of High Temperature and Water Stress on Pollen Germination and Spikelet Fertility in Rice. Environmental and Experimental Botany, 70, 58-65. https://doi.org/10.1016/j.envexpbot.2010.08.009

[9] Ding, C.Q., You, J., Chen, L., Wang, S.H. and Ding, Y.F. (2014) Nitrogen Fertilizer Increases Spikelet Number per Panicle by Enhancing Cytokinin Synthesis in Rice. Plant Cell Reports, 33, 363-371. https://doi.org/10.1007/s00299-013-1536-9

[10] Korres, N.E., Norsworthy, J.K., Burgos, N.R. and Oosterhuis, D.M. (2017) Temperature and Drought Impacts on Rice Production: An Agronomic Perspective Regarding Short- and Long-Term Adaptation Measures. Water Resources and Rural Development, 9, 12-27. https://doi.org/10.1016/j.wrr.2016.10.001

[11] Zhang, C.X., Feng, B.H., Chen, T.T., Zhang, X.F., Tao, L.X. and Fu, G.F. (2017) Sugars, Antioxidant Enzymes and IAA Mediate Salicylic Acid to Prevent Rice Spikelet Degeneration Caused by Heat Stress. Plant Growth Regulation, 83, 313-323. https://doi.org/10.1007/s10725-016-0232-5 
[12] Kamiji, Y., Yoshida, H., Palta, J.A., Sakuratani, T. and Shiraiwa, T. (2011) N Applications That Increase Plant N during Panicle Development Are Highly Effective in Increasing Spikelet Number in Rice. Field Crops Research, 122, 242-247. https://doi.org/10.1016/j.fcr.2011.03.016

[13] Tian, Q.L., Liu, B., Zhong, X.Y., Zhao, M., Sun, H. and Ren, W.J. (2016) Relationship of NSC with the Formation of Branches and Spikelets and the Yield Traits of Indica Hybrid Rice in Different Planting Methods. Scientia Agricultura Sinica, 1, 35-53.

[14] Zhang, B.L. and Yamagishi, J. (2010) Response of Spikelet Number per Panicle in Rice Cultivars to Three Transplanting Densities. Plant Production Science, 13, 279-288. https://doi.org/10.1626/pps.13.279

[15] Xu, F.X., Zhou, X.B., Zhang, L. and Jiang, P. (2018) Effects of Climatic Factors in the Southeast of Sichuan Basin on Grain Yield of Mid-Season Hybrid Rice. Scientia Agricultura Sinica, 4, 601-613. https://doi.org/10.3724/SP.J.1006.2018.00601

[16] Pal, R., Mahajan, G., Sardana, V. and Chauhan, B.S. (2017) Impact of Sowing Date on Yield, Dry Matter and Nitrogen Accumulation, and Nitrogen Translocation in Dry-Seeded Rice in North-West India. Field Crops Research, 206, 138-148. https://doi.org/10.1016/j.fcr.2017.01.025

[17] Bashir, M.U., Akbar, N., Iqbal, A. and Zaman, H. (2010) Effect of Different Sowing Dates on Yield and Yield Components of Direct Seeded Coarse Rice (Oryza sativa L.). Pakistan Journal of Agricultural Sciences, 47, 361-365.

[18] Zeng, Y.H., Zhang, Y.P., Wang, Y.L., Xiang, J., Chen, H.Z. and Zhu, D.F. (2015) Effects of Sowing Date on Formation of Branches and Spikelets in Indica-Japonica Hybrid Rice. Scientia Agricultura Sinica, 7, 13300-11310.

[19] Xing, Z.P., Cao, W.W., Qian, H.J., Hu, Y.J., Zhang, H.C., Dai, Q.G., et al. (2015) Effect of Sowing Date on Yield and Characteristics of Photosynthesis and Matter Production of Different Types in Mechanical Transplanted Rice. Journal of Nuclear Agricultural Sciences, 29, 528-537.

[20] Kato, Y., Kamoshita, A. and Yamagishi, J. (2008) Preflowering Abortion Reduces Spikelet Number in Upland rice (L.) under Water Stress. Crop Science, 48, 2389. https://doi.org/10.2135/cropsci2007.11.0627

[21] Ansari, T.H., Yamamoto, Y., Yoshida, T., Miyazaki, A. and Wang, Y. (2003) Cultivar Differences in the Number of Differentiated Spikelets and Percentage of Degenerated Spikelets as Determinants of the Spikelet Number per Panicle in Relation to Dry Matter Production and Nitrogen Absorption. Soil Science and Plant Nutrition, 49, 433-444. https://doi.org/10.1080/00380768.2003.10410029

[22] Van Oort, P.A.J., Balde, A., Diagne, M., Dingkuhn, M., Manneh, B., Muller, B., et al. (2016) Intensification of an Irrigated Rice System in Senegal: Crop Rotations, Climate Risks, Sowing Dates and Varietal Adaptation Options. European Journal of Agronomy, 80, 168-181. https://doi.org/10.1016/j.eja.2016.06.012

[23] Waha, K., Bussel, L.G.J., Müller, C. and Bondeau, A. (2012) Climate-Driven Simulation of Global Crop Sowing Dates. Global Ecology and Biogeography, 21, 247-259. https://doi.org/10.1111/j.1466-8238.2011.00678.x

[24] Maqueira López, L.A., Nova, W.T.D.L., Pérez Mesa, S.A., Díaz Paez, D. and Roján Herrera, O. (2016) Influence of Environmental Temperature and Sowing Date on the Phenological Phase Lengthening of Four Rice (Oryza sativa L.) Cultivars. Cultivos Tropicales, 37, 65-70.

[25] Xing, Z.P., Cao, W.W., Qian, H.J., Hu, Y.J., Zhang, H.C. and Dai, Q.G. (2016) Effect 
of Sowing Date on the Formation of Quality of Mechanically Transplanted Rice in Rice-Wheat Cropping Areas. Chinese Journal of Ecology, 1, 1-10.

[26] Hadgu, G., Tesfaye, K. and Mamo, G. (2015) Analysis of Climate Change in Northern Ethiopia: Implications for Agricultural Production. Theoretical and Applied Climatology, 121, 733-747. https://doi.org/10.1007/s00704-014-1261-5

[27] Pal, R., Mahajan, G., Sardana, V., Sharma, N. and Chauhan, B.S. (2018) Grain Quality of Dry-Seeded Rice in Response to Sowing Dates and Genotypes. International Journal of Plant Production, 12, 95-106. https://doi.org/10.1007/s42106-018-0010-6

[28] Espino, L., Way, M.O., Pearson, R. and Nunez, M. (2009) Effect of Planting Date on Lissorhoptrus oryzophilus (Coleoptera: Curculionidae) Density-Yield Relationship on Rice in Southeastern Texas. Journal of Economic Entomology, 102, 1536. https://doi.org/10.1603/029.102.0418

[29] Ke, X., Sun, Z., Huo, Z.Y., Dai, Q.G., Zhang, H.C., Liu, J., et al. (2013) Effects of Seeding Date and Variety Type on Yield, Growth Stage and Utilization of Temperature and Sunshine in Rice. Scientia Agricultura Sinica, 46, 4222-4233.

[30] Ahmed, S., Humphreys, E., Salim, M. and Chauhan, B.S. (2014) Optimizing Sowing Management for Short Duration Dry Seeded Aman Rice on the High Ganges River Floodplain of Bangladesh. Field Crops Research, 169, 77-88. https://doi.org/10.1016/j.fcr.2014.09.009

[31] Ma, S.C., Wang, T.C., Guan, X.K. and Zhang, X. (2018) Effect of Sowing Time and Seeding Rate on Yield Components and Water Use Efficiency of Winter Wheat by Regulating the Growth Redundancy and Physiological Traits of Root and Shoot. Field Crops Research, 221, 166-174. https://doi.org/10.1016/j.fcr.2018.02.028

[32] Fu, G.F., Zhang, C.X., Yang, X.Q. and Yang, Y.J. (2015) Action Mechanism by Which SA Alleviates High Temperature Induced Inhibition to Spikelet Differentiation. Chinese Journal of Rice Science, 29, 637-647.

[33] Yoshida, H., Horie, T. and Shiraiwa, T. (2006) A Model Explaining Genotypic and Environmental Variation of Rice Spikelet Number per Unit Area Measured by Cross-Locational Experiments in Asia. Field Crops Research, 97, 337-343. https://doi.org/10.1016/j.fcr.2005.11.004

[34] Biswas, P.K. and Salokhe, V.M. (2001) Effects of Planting Date, Intensity of Tiller Separation and Plant Density on the Yield of Transplanted Rice. The Journal of Agricultural Science, 137, 279-287. https://doi.org/10.1017/S0021859601001307

[35] Sha, X.Y. and Linscombe, S.D. (2007) Planting Date Affects Grain and Milling Yields of Water-Seeded Clearfield Rice. Agronomy Journal, 99, 1143-1150. https://doi.org/10.2134/agronj2006.0298

[36] Wang, Y.X., Yang, L.X., Kobayashi, K., Zhu, J.G., Chen, C.P., Yang, K.F., et al. (2012) Investigations on Spikelet Formation in Hybrid Rice as Affected by Elevated Tropospheric Ozone Concentration in China. Agriculture Ecosystems \& Environment, 150, 63-71. https://doi.org/10.1016/j.agee.2012.01.016

[37] Kobayasi, K., Yamane, K. and Imaki, T. (2001) Effects of Non-Structural Carbohydrates on Spikelet Differentiation in Rice. Plant Production Science, 4, 9-14. https://doi.org/10.1626/pps.4.9

[38] Zhong, L., Chen, X.R., Hu, H.J., Wang, Q.H., Zheng, X.W., Ren, F.Z., et al. (2007) Genotypic Difference and the Classification in Response of Differentiation and Retrogression of Branch and Spikelet to Seeding-Date in Different Hybrid Rice Parents. Acta Agriculturae Universitatis Jiangxiensis, 5, 695-700. 